\title{
MACC1 induces autophagy to regulate proliferation, apoptosis, migration and invasion of squamous cell carcinoma
}

\author{
JIAN WU ${ }^{1 *}$, DAWEI ZHANG ${ }^{1 *}$, JUN LI $^{1}$, XIN DENG $^{3,4}$, GUANNAN LIANG $^{1}$, \\ YANG LONG ${ }^{1}$, XUEMEI HE ${ }^{1}$, TIANYANG DAI ${ }^{1}$ and DELIAN REN ${ }^{2}$ \\ ${ }^{1}$ Department of Cardio-Thoracic Surgery, The Affiliated Hospital of South West Medical University; \\ ${ }^{2}$ Department of Immunology, Basic Medicine College, South West Medical University; \\ ${ }^{3}$ Drug Discovery Research Center, Southwest Medical University; ${ }^{4}$ Laboratory for Cardiovascular Pharmacology of \\ Department of Pharmacology, The School of Pharmacy, Southwest Medical University, Luzhou, Sichuan, P.R. China
}

Received January 21, 2017; Accepted June 12, 2017

DOI: $10.3892 /$ or.2017.5889

\begin{abstract}
Metastasis-associated colon cancer-1 (MACC1) plays an important role in cancer development, but the role and mechansim of MACC1 in squamous cell carcinoma (ESCC) remain unclear. In this study, we found that MACC1 expression was increased in ESCC, and correlated with lymph node metastasis. MACC1 knockdown suppresed ESCC cell proliferation, metastasis and enchanced cell apoptosis. Moreover, MACC1 knockdown inhibited ESCC cell autophagy, and 3-methyladenine was able to rescue MACC1induced malignant phenotype of ESCC cells. Furthermore, MACC1 knockdown inactivated AMPK-ULK1 signaling pathway, and metformin could rescue MACC1-induced autophagy in ESCC cells. Collectively, this study found that upregulation of MACC1 in ESCC was associated with lymph node metastasis of patients, and MACC1 regulated ESCC cell proliferation, apoptosis, migration and invasion mainly through AMPK-ULK1 induced autophagy.
\end{abstract}

\section{Introduction}

Esophageal cancer (ESC) is a common malignant tumor in digestive tract system, and its incidence and mortality rank eighth and sixth wordwide, respectively $(1,2)$. ESC mainly includes two subtypes: squamous cell carcinoma (ESCC) and adenocarcinoma. The former accounts for $90 \%$ of patients with ESC in China (3). In recent years, although the clinical treatment for ESC has improved, the 5-year survival rate of patients

Correspondence to: Professor Delian Ren, Department of Immunology, Basic Medicine College, South West Medical University, Luzhou, Sichuan 646000, P.R. China

E-mail: rendelian0605@sina.com

*Contributed equally

Key words: metastasis-associated colon cancer-1, metastasis, autophagy, AMPK signaling, esophageal squamous cell carcinoma with ESC is still between 15 and $25 \%$, and this is strongly associated with the high recurrence and metastasis $(4,5)$. Most of the patients with ESC have advanced stage disease when diagnosed, because the early stage of ESC is difficult to discover (6).

Metastasis-associated colon cancer-1 (MACC1), a tumor metastasis-related gene, was first identified in colon cancer by Stein et al in 2009 (7). Its encoding proteins include ZU5, SH3 binding domain, SH3 structural domain, and two death domains of the C-terminal tail (8). MACC1 binds sp1 and further locates in the promoter region of c-Met gene to promote the gene transcription, thereby enhancing the proliferation and metastasis of colon cancer cells (9). Recent studies indicated that upregulation of MACC1 promoted cell proliferation and metastasis in various cancers, such as gastric cancer $(10,11)$, hepatocellular carcinoma $(12,13)$, breast cancer $(14)$, ovarian cancer (15) and glioma (16). However, the role of MACC1 in ESC development remains largely unclear.

Autophagy refers to a highly conserved biological process in which cytoplasmic materials are delivered to lysosomes for degradation and reuse, and this process plays an important role in tumor recurrence, drug resistance, and distant metastasis (17-19). Increasing evidence has shown that deficiency of key autophagy-related genes induces tumorigenesis and enhances survival ability of tumor cells $(20,21)$. On the contrary, accumulated autophagy causes irreversible damage to organelles, resulting in tumor cell death (22). Autophagy is a double-edged sword in tumor progress, but it still provides a new strategy for treatment of tumor. In the present study, we found that MACC1 overexpression induced autophagy to promote ESC cell proliferation, migration and invasion, suggesting that MACC1 might be a theraputic target for the treatment of ESC.

\section{Materials and methods}

Clinical specimens. Samples were collected from 51 patients with ESCC, who underwent complete surgical resection at Department of Cardiothoracic Surgery of the Affiliated Hospital of Southwest Medical University from March 2011 to December 2014. This study was approved by Ethics Review 
Board at Southwest Medical University. After surgical resection, the tumor and matched normal tissues (away from the tumor by $>5 \mathrm{~cm}$ ) were isolated and stored in liquid nitrogen. Clinical characterization of the patients with ESCC are shown in Table I.

Immunohistochemistry. Any five high-power fields (x200) were selected for measurement, and density of the MACC1 staining was scored based on two pathological experts. The scores were as follows: 0 for no color, 1 for light yellow, 2 for yellow, and 3 for brown. Percentage of positive cells was calculated under the view, and scoring was performed according to the following standards: $\leq 5 \%$, a score of $0 ; 6-25 \%$, a score of 1 ; $26-50 \%$, a score of 2; and $51-100 \%$, a score of 3 . The final score was obtained by multiplying the average staining intensity of each slice by the average percentage of positive cells, with 0-1 score for negative (-), 2-4 for weakly positive (+), and 5-9 for strongly positive (++).

Cell culture. Normal human esophageal epithelial cells, and esophageal squamous cell carcinoma cell lines including Eca109, Eca9706, TE1, TE10, TE11 and Kyse150 were cultured in vitro. Normal esophageal epithelium cells were cultured using EpiCGS-2 serum-free epithelial medium containing $1 \% \mathrm{P} / \mathrm{S}$ solution, and esophageal squamous cell carcinoma cells were cultured in RPMI-1640 medium supplemented with $10 \%$ fetal bovine serum (FBS) at $37^{\circ} \mathrm{C}$ in $5 \% \mathrm{CO}_{2}$, respectively.

RNA isolation. Tissues were quickly frozen by liquid nitrogen and completely pulverized. Tissue powder or ESC cells were lysated by using TRIzol isolation reagent according to the manufacturer's protocol. Total RNAs were analyzed using gel electrophoresis and ultraviolet spectrophotometer, and then stored at $-80^{\circ} \mathrm{C}$.

Quantitative reverse transcription polymerase chain reaction (qRT-PCR). PrimeScript RT reagent kits (Takara) were used to synthesize cDNA, and SYBR Green Real-time PCR Master Mix (Takara) was used to perform the PCR assays accoding to the manufacturer's protocols, respectively. Parameter of PCR reaction was as follows: incubation at $94^{\circ} \mathrm{C}$ for $2 \mathrm{~min}$, followed by 40 cycles of $94^{\circ} \mathrm{C}$ for $30 \mathrm{sec}, 60^{\circ} \mathrm{C}$ for $1 \mathrm{~min}$ and $72^{\circ} \mathrm{C}$ for $30 \mathrm{sec}$, and after that incubation at $72^{\circ} \mathrm{C}$ for $2 \mathrm{~min}$. The sequences of primers of MACC1 and GAPDH are as follows: MACC1 forward, 5'-GCAGACAAAGAATCAGAGAAAG-3'; reverse, 5'-GATGAGACGTGCGACTAACTC-3'.GAPDH forward, 5'-ATGCTGGCGCTGAGTACGTC-3'; reverse, 5'-GGTCATGAGTCCTTCCACGATA-3'.

Western blotting. Total proteins were extracted using RIPA lysis buffer (Pierce Biotechnology) according to the manufacturer's protocol. Forty micrograms of total proteins were separeted on $10 \%$ SDS-PAGE gels and then transferred onto PVDF membranes. The membranes were blocked in five percent skimmed milk for $1 \mathrm{~h}$, and then incubated with primary antibodies, MACC1 (1:1,000, Sigma) and GAPDH (1:4000, Beyotime), at $4^{\circ} \mathrm{C}$ overnight. The membranes were incubated with HRP-conjugated secondary antibodies (Zhongshan Biotechnology) for $1 \mathrm{~h}$, and finally analyzed by using enhanced chemiluminescence.
Table I. Clinical characterization of patients with ESCC.

Index

Sex

Male

Female

Age

$>60$

$\leq 60$

Tumor size

$>5 \mathrm{~cm}$

$\leq 5 \mathrm{~cm}$

Location

Middle

Lower

Differentiation

Well

Moderate

Poor

Invasion depth

Outer layer

Inner layer

Lymph node metastasis

Yes

No

Lentivirus vector construct. Eca9706 and Kyse150 cells were inoculated in six-well plates with a density of $2 \times 10^{5}$ cells per well. After the cells adhered to the plates, lentivirus LV-GFPPURO-shR-MACC1 and LV-GFP-PURO-shR-NC were added to infect with the multiplicity of infection (MOI) value of 20 . After $12 \mathrm{~h}$, fresh complete RPMI-1640 medium was replaced. At $72 \mathrm{~h}$ post medium replacement, complete RPMI-1640 medium containing $1 \mu \mathrm{g}$ of PURO was added to the culture for 14 days. Efficiency of transfection was measured by immunofluorescence microscopy and flow cytometry. Silencing effect of MACC1 was measured by western blotting.

Cell proliferation. ESCC cells with a density of $1 \times 10^{4}$ per well were seeded into 96-well plates. When the cells were cultured for 24,48 or $72 \mathrm{~h}$, cell proliferation was analyzed using cell counting kit 8 (CCK8, Beyotime) according to the manufacturer's protocol.

Cell migration and invasion. For cell migration, $1 \times 10^{5}$ ESCC cells were resuspended in $200 \mu \mathrm{l}$ serum-free RPMI-1640 medium and added to the upper compartment of Transwell chambers $(8 \mu \mathrm{m})$, which were placed into 24 -well plates. RPMI-1640 medium (500 $\mu \mathrm{l}$ ) supplemented with 10\% FBS was added into each well of the 24-well plates, so that it could be attracted to the lower compartment of Transwell chambers, and then incubation at $37^{\circ} \mathrm{C}$ with $5 \% \mathrm{CO}_{2}$ for $24 \mathrm{~h}$. The chambers were fixed with $4 \%$ formaldehyde at room temperature for $10 \mathrm{~min}$, and then stained with Giemsa for $2 \mathrm{~min}$. The cells detained in the upper compartment were removed, and the 

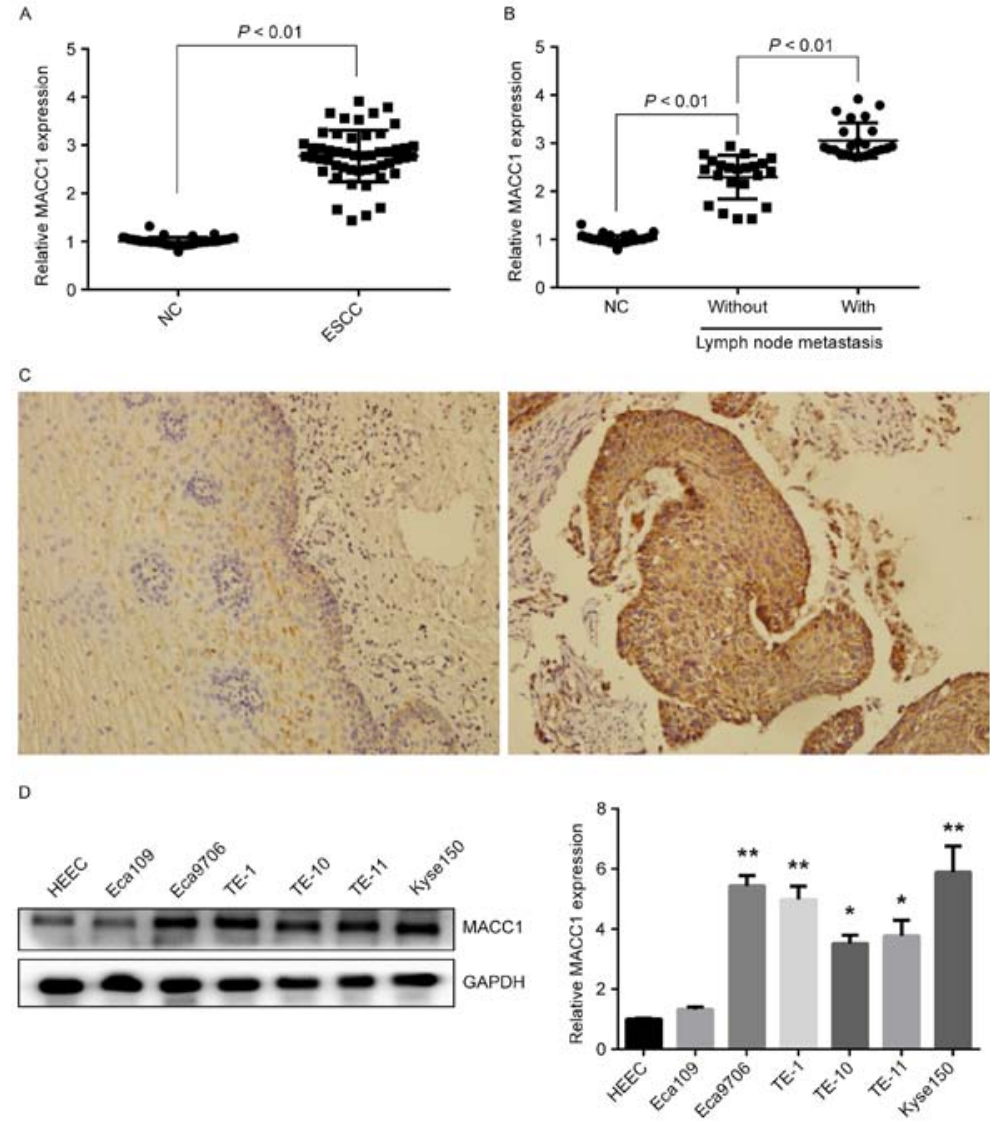

Figure 1. MACC1 expression in ESCC tissues and cell lines. QRT-PCR revealed increased MACC1 expression in 51 ESCC tissues compared to paired normal tissues (A), and MACC1 expression was increased in 29 ESCC tissues with lymph node metastasis and in 22 of those without (B). Immunohistochemical staining reveals that MACC1 expression is increased in ESCC tissues (C). Western blotting and qRT-PCR show MACC1 expression in ESCC cell lines (D). $\beta$-actin as internal reference. ${ }^{* *} \mathrm{P}<0.01 ;{ }^{*} \mathrm{P}<0.05$.

cells passed through the membrane were observed under a microscope. Five fields were randomly selected and the cells were counted.

For cell invasion, the procedure was the same, except that Matrigel (CA, USA) was added into the upper chamber of Transwell chambers to simulate extracellular matrix, and the incubation time continued for $72 \mathrm{~h}$. Briefly, the original Matrigel was diluted with serum-free RPMI-1640 medium at a ratio of $1: 2$. Diluted Matrigel $(50 \mu \mathrm{l})$ was added evenly to coat the upper chamber, and incubated at $37^{\circ} \mathrm{C}$ for $60 \mathrm{~min}$.

Cell apoptosis. The cells were collected and washed twice with pre-cooled PBS. Cell apoptosis was analyzed using Annexin V Fluorescein Isothiocyanate Apoptosis Detection kit (BD Bioscience) according to the manufacturer's protocol. The single PE-Annexin V-positive population and double-positive [PE and 7-amino-actinomycin D (AAD)] population detected by flow cytometry were considered as early and late apoptotic cells, respectively. The single PI-7AAD-positive population was considered as necrotic cells.

Confocal laser scanning microscopy and transmission electron microscopy. For confocal laser scanning microscopy, $1 \times 10^{5}$ cells in each group were inoculated on a $20-\mathrm{mm}$ culture dish and cultured in RPMI medium supplemented with $10 \%$ FBS. After the GFP-RFP-LC3B adenovirus
(MOI, 20; purchased from Han Bio) was added in the culture medium and co-cultured for $12 \mathrm{~h}$, the medium was replaced by a fresh medium and then cultured for $72 \mathrm{~h}$. Imagings were observed under a confocal fluorescence microscope (SP8, Leica, Germany). The numbers of autophagosomes (green) and autolysosomes (red) in random field were calculated.

For transmission electron microscopy, growing adherent cells were directly scraped, and the cell precipitate was collectet after using PBS to wash twice. The cells were fixed by using $2.5 \%$ glutaraldehyde solution overnight at $4^{\circ} \mathrm{C}$. Two days later, imagings were observed under a transmission electron microscope (Fucheng Biological Technology Co., Ltd., Shanghai, China).

Statistical analyses. Continuous data are presented as mean \pm standard deviation. The comparison between two groups was performed by using Student's t-test. A P $<0.05$ was considered statistically significant. All experiments were repeated three times.

\section{Results}

MACC1 is frequently upregulated in ESCC tissues and associated with lymph node metastasis. To determine MACC1 expression in ESCC, we detected mRNA and protein expressions of MACC1 in 51 pairs of ESCC and matched normal 
A
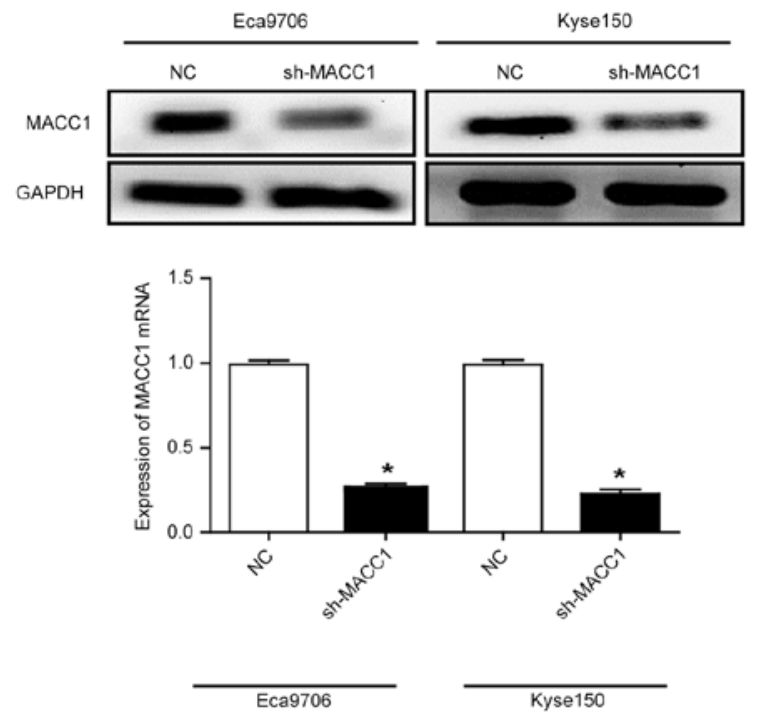
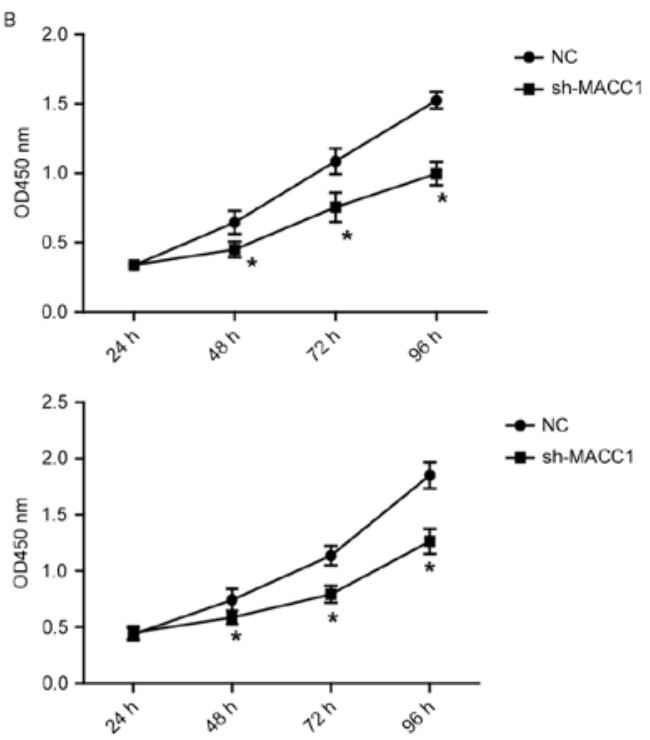

c

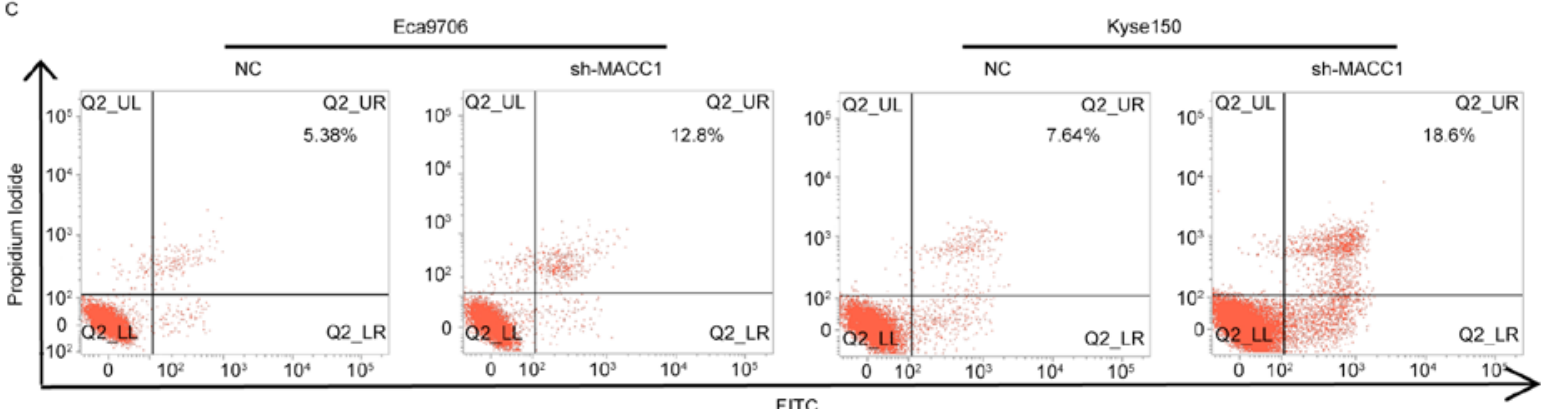

Figure 2. MACC1 regulates ESCC cell proliferation and apoptosis. (A) Western blotting (upper) and qRT-PCR (lower) revealed MACC1 expression is decreased in the Eca9706 and Kyse150 cells infected with lentivirus containing sh-MACC1 vectors, compared with those containing empty vectors. (B) Cell count kit-8 shows MACC1-knockdwon Eca9706 and Kysel150 cells have decreased proliferative capacity after culture for 48, 72 and 96 h. (C) Flow cytometry showed MACC1-knockdown Eca9706 and Kysel150 cells have increased apoptosis rates. " $\mathrm{P}<0.05$.

A
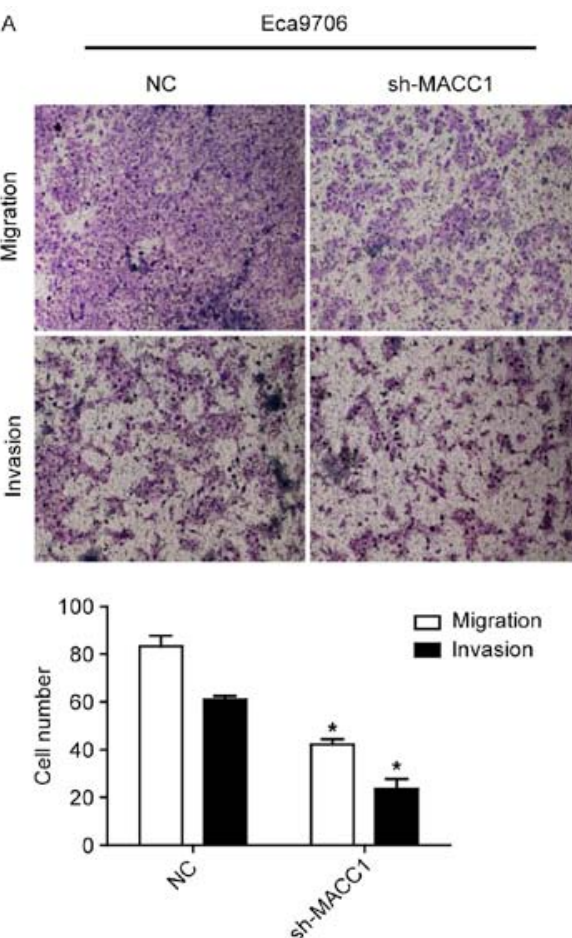

B
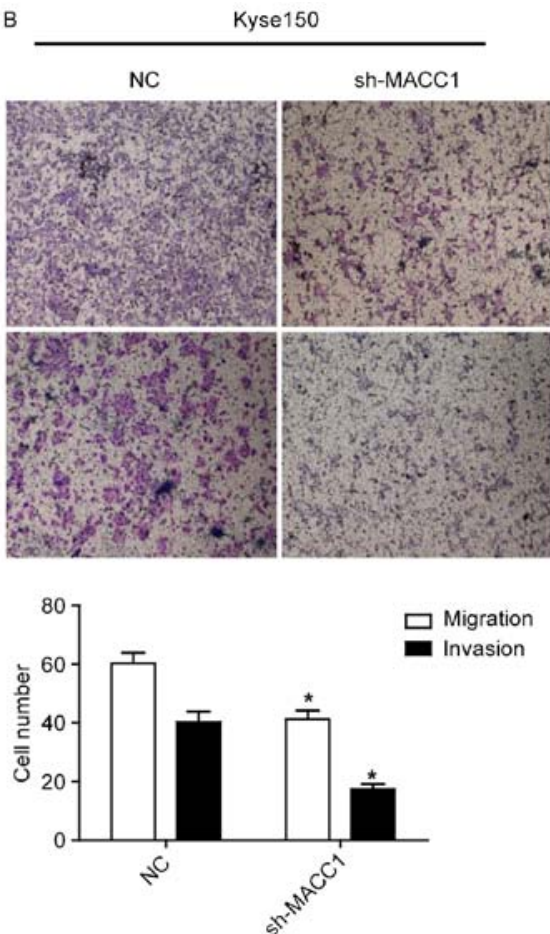

Figure 3. MACC1 knockdown inhibits ESCC cells migration and invasion. (A) Transwell without Matrigel shows that MACC1-knockdown Eca9706 and Kysel150 cells have a decreased migratory capacity. (B) Transwell with Matrigel shows that MACC1-knockdown Eca9706 and Kysel150 cells have a decreased invasive capacity. 


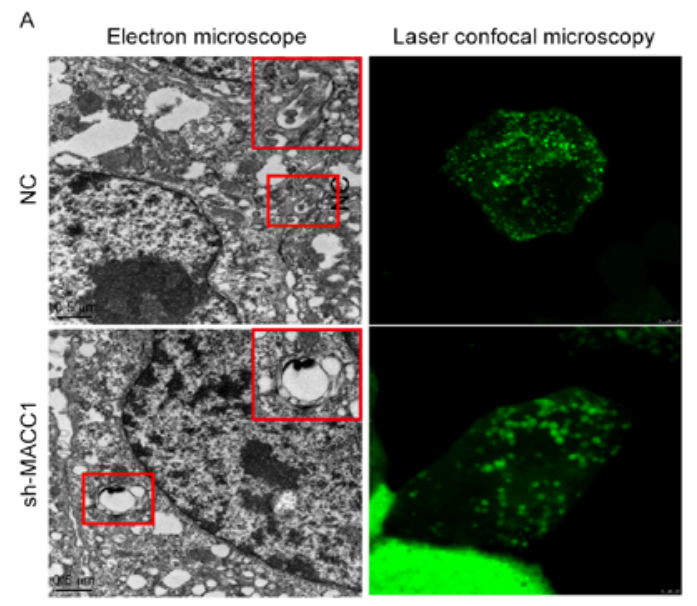

B

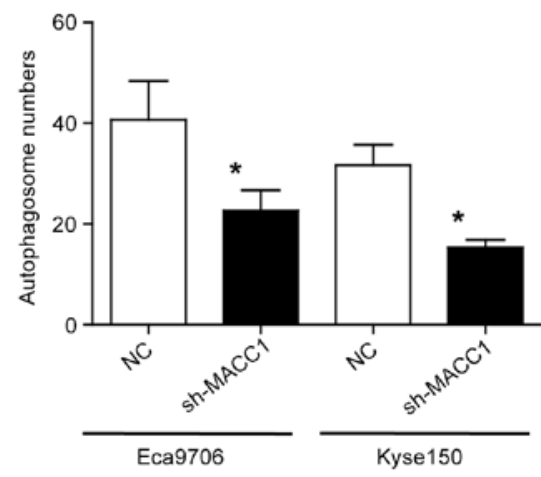

C

D
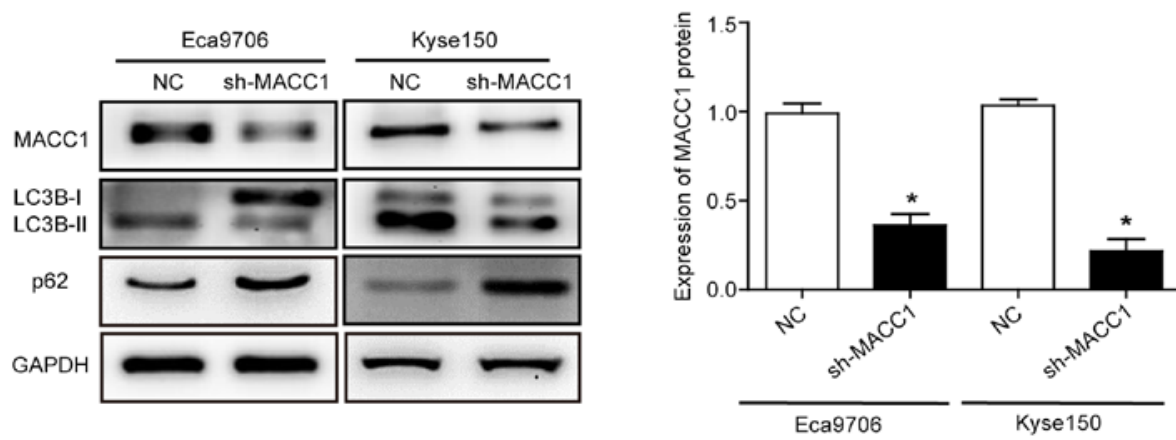

E
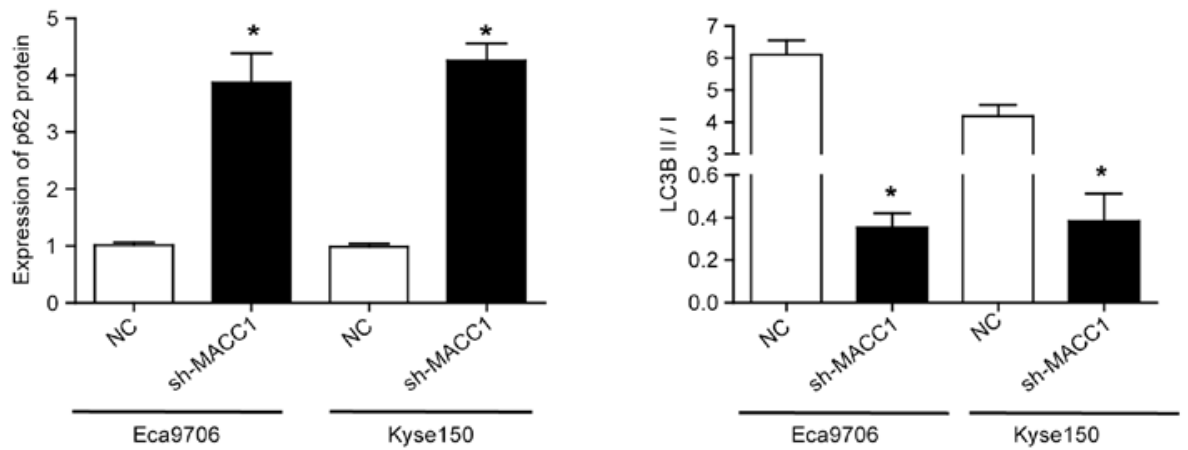

Figure 4. MACC1 knockdown inhibits ESCC cell autophagy. (A) Electron microscope and laser confocal microscopy show autophagy in Eca9706 cells. (B) The number of autophagosome is decreased in MACC1-knockdown Eca9706 and Kyse150 cells. (C) Western blotting revealed that MACC1 knockdown decrease MACC1 expression (D), and the ratio of LC3B-I/II and increases p62 expression (E) in the Eca9706 and Kyse150 cells. * $\mathrm{P}<0.05$.

tissues. QRT-PCR experiments showed the mRNA expression of MACC1 was significantly increased in ESCC tissues compared with matched normal tissues $(\mathrm{P}<0.01)$ (Fig. 1A), and the expression was also increased in ESCC tissues of patients with lymph node metastasis compared to those without lymph node metastasis $(\mathrm{P}<0.01)$ (Fig. 1B). Immunohistochemical staining revealed that the protein expression of MACC1 was also increased in ESCC tissues with $94.1 \%$ (48/51) positive rate compared with matched normal tissues with $37.3 \%(19 / 51)$ positive rate (Fig. 1C). Furthermore, mRNA and protein expressions of MACC1 in ESCC cell lines were examined. Western blotting and qRT-PCR assays showed high protein and mRNA expression of MACC1 in Eca9706 and Kyse150 cells $(\mathrm{P}<0.01)$ (Fig. 1D), suggesting that MACC1 expression is increased in ESCC and associated with lymph node metastasis of ESCC patients.
MACC1 regulates proliferation and apoptosis of ESCC cells. To determine the role of MACC1 in ESCC cells, we selected Eca9706 and Kyse150 cells with high expression of MACC1 to perform MACC1 knockdown using letivirus vectors containing the specific shRNA for MACC1. Western blotting and qRT-PCR assays showed that the protein and mRNA expression of MACC1 was significantly decreased, respectively $(\mathrm{P}<0.05)$ (Fig. 2A). Cell count kit-8 experiments indicated that MACC1 knockdown significantly decreased proliferative capacity of the Eca9706 and Kyse150 cells $(\mathrm{P}<0.05)$ (Fig. 2B). Flow cytometry revealed that MACC1 knockdown significantly increased apoptosis of the Eca9706 and Kyse150 cells (Fig. 2C).

Downregulation of MACC1 suppreses migration and invasion of ESCC cells. To determine whether MACC1 is involved in ESCC cell metastasis, cell migration and invasion assays were 

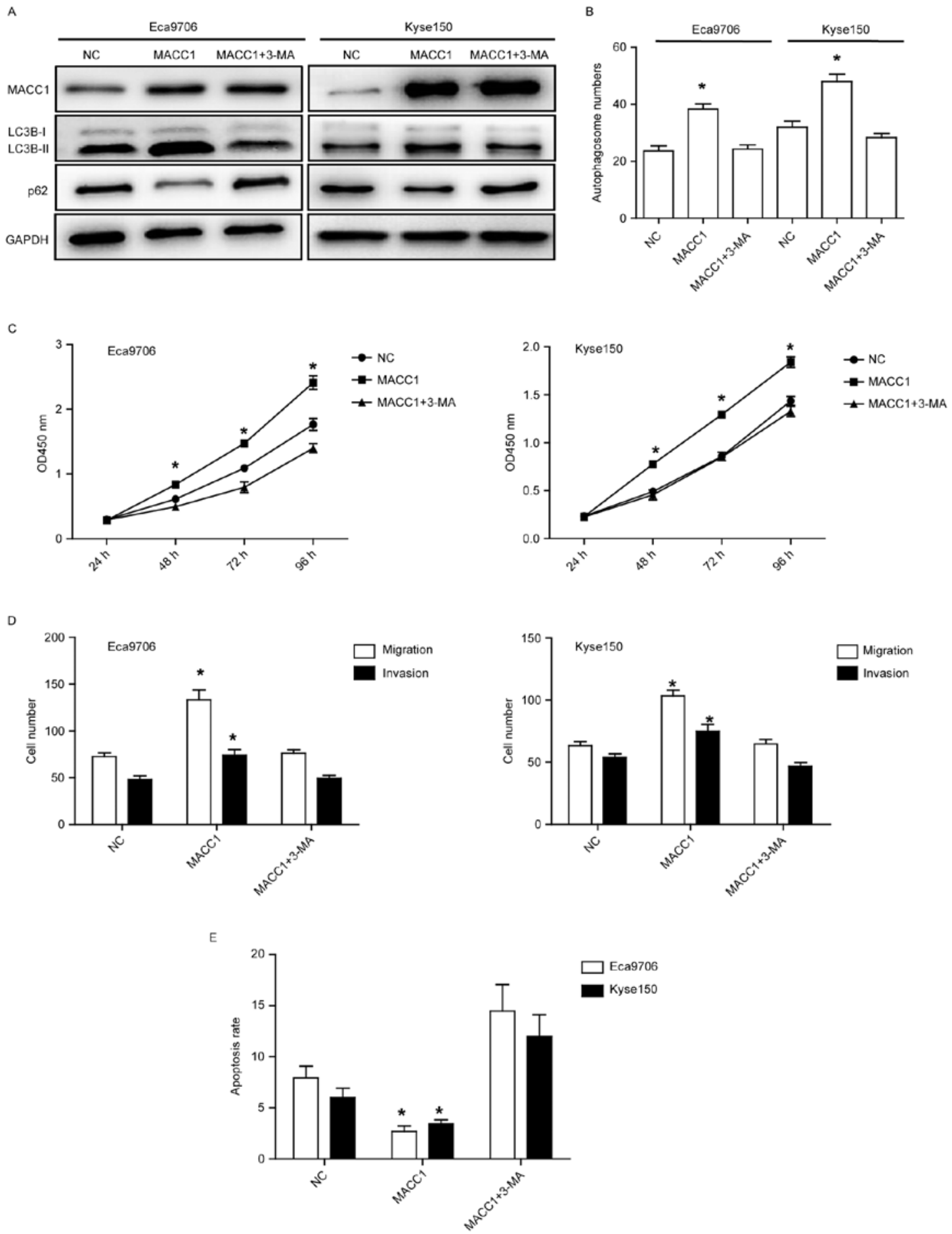

Figure 5. Three-methyladenine (3-MA) rescues MACC1-induced ESCC cell phenotype. (A) Western blotting shows that treatment of 3-MA decreases the ratio of LC3B-I/II and increases p62 expression in Eca9706 and Kyse150 cells with MACC1 overexpression. (B) Number of autophagosomes in the Eca9706 and Kyse150 cells was analyzed after 3-MA treatment. (C) Cell counting kit-8 reveals that treatment of 3-MA rescues the proliferation of Eca9706 and Kyse150 cells at 48, 72 and $96 \mathrm{~h}$. (D) Transwell reveals that treatment of 3-MA rescues the migration and invasion of MACC1-overexpressing Eca9706 and Kyse150 cells. (E) Flow cytometry shows that treatment of 3-MA rescues apoptosis of MACC1-overexpressing Eca9706 and Kyse150 cells. "P<0.05.

performed. As shown in Fig. 3, MACC1 knockdown significantly repressed migration and invasion of the Eca9706 and Kyse150 cells $(\mathrm{P}<0.05)$, suggesting that $\mathrm{MACC} 1$ is correlated with tumor metastasis in ESCC.

Downregulation of MACC1 inhibits autophagy in ESCC cells. Autophagy is shown to be involved in the proliferation, apoptosis and metastasis of tumor cells. Therefore, we determined whether MACC1 is involved in ESCC cell autophagy. Electron microscope and laser confocal microscopy assays showed autophagy (Fig. 4A), and the number of autophagosome was significantly decreased in the Eca9706 and Kyse150 cells with downregulating MACC1 compared with those with empty vector $(\mathrm{P}<0.05)$ (Fig. 4B). Moreover, western blotting revealed that MACC1 knockdown significantly decreased the ratio of LC3B II/I $(\mathrm{P}<0.05)$ and increased the protein expression of 

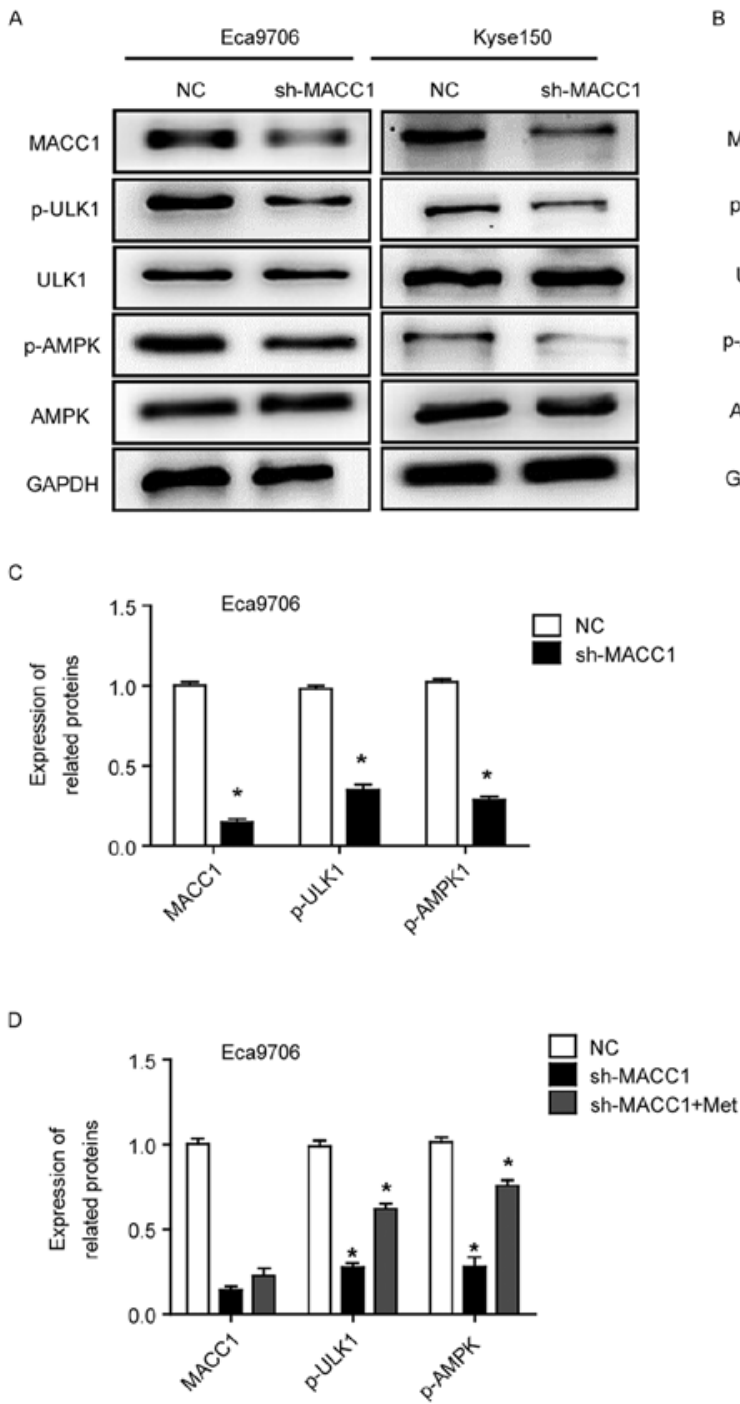

B
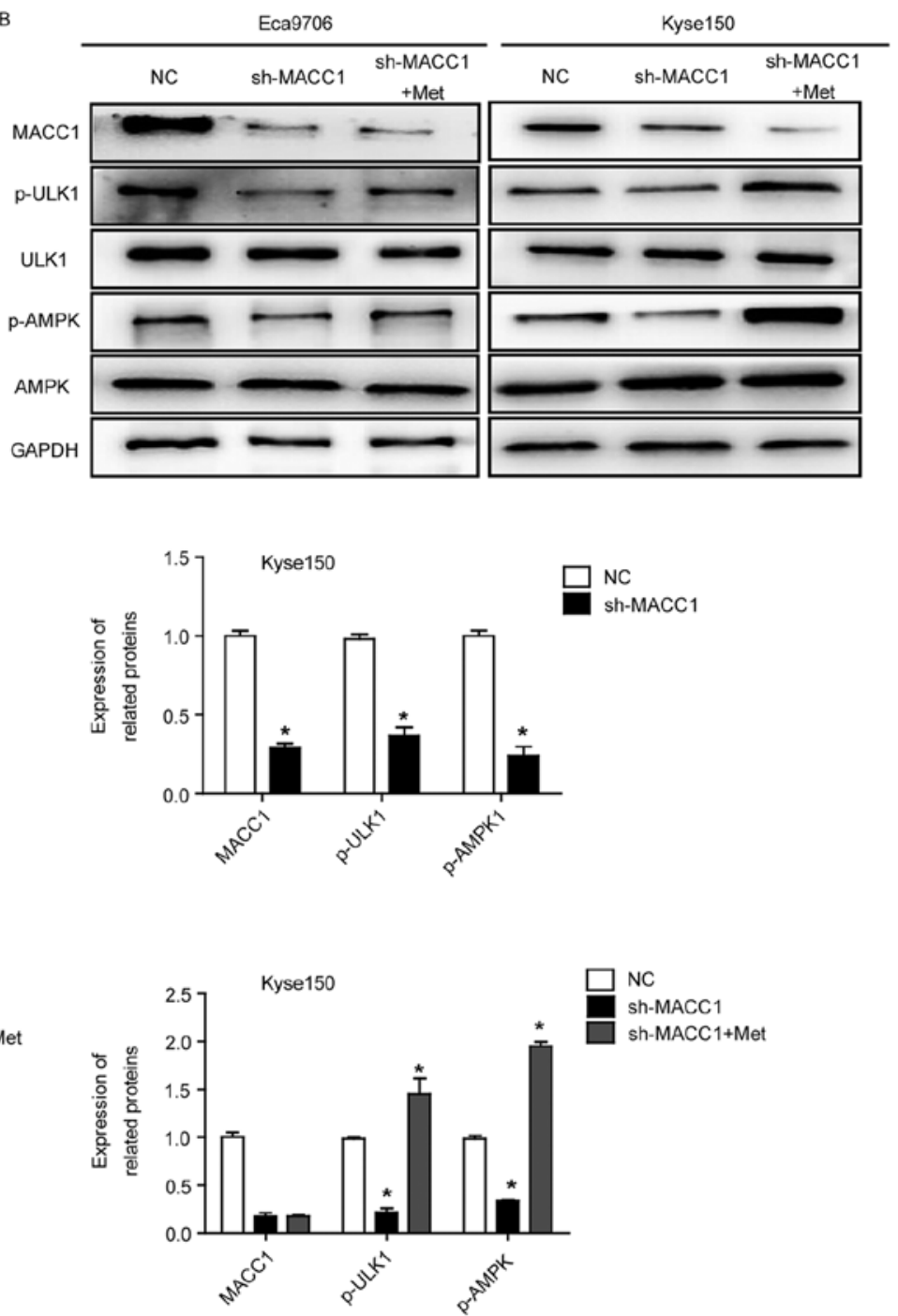

Figure 6. MACC1 regulates AMPK signaling pathway. (A and C) Western blotting shows MACC1 knockdown decreased phosphorylation levels of ULK1 and AMPK in Eca9706 and Kyse150 cells. (B and D) Western blotting reveals that treatment of metformin rescues the phosphorylation levels of ULK1 and AMPK in the Eca9706 and Kyse150 cells. * $\mathrm{P}<0.05$.

p62 $(\mathrm{P}<0.05)$ (Fig. 4C-E). These data suggest that MACC1 knockdown inhibits autophagy in ESCC cells.

Three-methyladenine (3-MA) rescues MACC1-induced ESCC cell proliferation, apoptosis, migration, and invasion. To detemine whether autophagy plays a crucial role in MACC1induced phenotype in ESCC cells, we used 3-methyladenine (3-MA), an inhibitor of autophagy, to inhibit autophagy in Eca9706 and Kyse150 cells. Western blotting and laser confocal microscopy assays showed that 3-MA rescued the ratio of LC3B II/I and p62 expression, and the number of autopahosome, respectively $(\mathrm{P}<0.05)$ (Fig. 5A and $\mathrm{B}$ ), suggesting that MACC1-induced autophagy was rescued by 3-MA treatment. Furthermore, proliferation, apoptosis, migration, and invasion of the Eca9706 and Kyse150 cells were also rescued with 3-MA treatment $(\mathrm{P}<0.05)$ (Fig. 5C-E), suggesting MACC1-induced phenotype in ESCC cells is mainly through autophagy.

MACC1 regulates the AMPK signaling pathway involved in autophagy. To understand the mechanism by which MACC1 regulates ESCC cell autophagy, we detected AMPK-ULK1 signaling pathway that is believed to be involved in cell autophagy. Western blot assays showed that MACC1 knockdown significantly decreased phosphorylation levels of p-ULK1 and p-AMPK in the Eca9706 and Kyse150 cells $(\mathrm{P}<0.05)$ (Fig. 6A and $\mathrm{C}$ ), whereas protein expression of ULK1 and AMPK was not altered, suggesting that MACC1-indued autophagy is associated with AMPK signaling pathway. Furthermore, MACC1-induced autophagy in ESCC cells was rescued when the cells were treated with metformin, an AMPK activator $(\mathrm{P}<0.05)$ (Fig. 6B and $\mathrm{D})$, suggesting that AMPK signaling pathway is a key pathway in MACC1induced autophagy.

\section{Discussion}

This study found that MACC1 was overexpressed in ESCC, and its upregulation was positively correlated with lymph node metastasis of ESCC patients. Stable knockdown of MACC1 repressed ESCC cell proliferation, migration and invasion, while enhanced cell apoptosis. Furthermore, 
MACC1 knockdown inhibited ESCC cell autophagy. When 3-MA, an inhibitor for autophagy, was used to treat ESCC cells, MACC1-induced autophagy was rescued, resulting in ESCC cell proliferation, apoptosis, migration, and invasion also being rescued to normal level, suggesting that MACC1 induced the aggressive progress of ESCC cells mainly through affecting autophagy. AMPK signaling pathway was also confirmed to be important in MACC1-induced autophagy.

MACC1 has been considered as an important molecule for various cancer diagnosis, prognosis, and theraputic target (10-15). Increasing evidence indicates that ectopic expression of MACC1 is involved in tumorigenesis and cancer development (16). However, the role and mechnism of MACC1 in ESCC had not been investigated. Our data demonstrated that MACC1 was upregulated in ESCC and correlated with lymph node metastasis of ESCC patients, suggesting that the expression signature of MACC1 might be a novel biomarker for ESCC diagnosis and prognosis. The role of MACC1 was reported inconsistently in variety of cancers, but in our data we first showed MACC1 as an oncogene, promoting malignant phenotypes of ESCC cells by gain- and loss-of-function.

Autophagy plays crucial role in tumorigenesis and cancer development. For example, Beclin deficience could increase the tumorigenesis risk of mice, such as lymphoma, lung cancer, liver cancer and other tumors $(23,24)$ Weh et al found that Beclin-dependent autophagy is specific and related with prognostic factors of EAC (25). Lee et al demonstrated that a dominant-negative autophagy protein Atg5K130R could lead to loss-of-heterozygosity of tp53 followed by promoting tumorigenesis (26). In our study, we first found that MACC1 induced ESCC cell autophagy by multiple experiments. Importantly, 3-MA, an inhibitor for autophagy, was able to rescue autophagy in ESCC cells, and further rescued MACC1-induced malignant phenotype of ESCC cells, suggesting the important role of autophagy in ESCC cells. However, 3-MA did not alter MACC1 expression, indicating that MACC1 is a upstream gene in autophagy of ESCC cells. Consistant with a previous study (27), our data also revealed that autophagy inhibition repressed ESCC cell proliferation, migration and invasion, and enhanced cell apoptosis. Autophagy is involved in several signaling pathways, such as AMPK and PI3K/ AKT/mTOR $(28,29)$. Our results showed that MACC1 knockdown decreased phosphorylation levels of AMPK and ULK1, suggesting that MACC1-induced autophagy is involved in AMPK signaling pathway. AMPK directly phosphorylates VPS34 and ULK1, resulting in autophagy formation. On the contrary, AMPK inhibits autophagy by phosphorylating mTOR, so AMPK has different roles in different cells. Moreover, when ESCC cells were treated using metformin, an activator of AMPK, the autophagy was rescued, suggesting MACC1-induced autophagy mainly through regulating AMPK signaling pathway.

In conclusion, the present study showed that upregulation of MACC1 was correlated with lymph node metastasis of ESCC patients. MACC1 regulated ESCC cell proliferation, apoptosis, migration and invasion mainly through affecting autophagy.

\section{Acknowledgements}

The authors wish to thank Mr. Biao Zhou, and KaiMing He for assistance in obtaining patient tissue samples. This study was supported by The Joint Fund of Technology Department, Sichuan Province (2014TSX-0102), and by Youth Foundation of Affiliated Hospital of South West Medical University (16025), and by Sichuan Science and Technology Plan projects (no. 2016RZ0076).

\section{References}

1. Sawada G, Niida A, Uchi R, Hirata H, Shimamura T, Suzuki Y, Shiraishi Y, Chiba K, Imoto S, Takahashi Y, et al: Genomic landscape of esophageal squamous cell carcinoma in a Japanese population. Gastroenterology 150: 1171-1182, 2016.

2. Mao Y, Li L, Liu J, Wang L and Zhou Y: miR-495 inhibits esophageal squamous cell carcinoma progression by targeting Akt1. Oncotarget 7: 51223-51236, 2016.

3. Wang HY, Yao ZH, Tang H, Zhao Y, Jin SL, Zhou WP, Yao SN, Yang SJ, Liu YY and Luo SX: A retrospective clinical study of comparing paclitaxel plus S-1 versus paclitaxel plus cisplatin as the first-line treatment for patients with advanced esophageal squamous cell carcinoma. Oncotarget 8: 7540-7547, 2017.

4. Hu D, Lin X, Chen Y, Chang Q, Chen G, Li C, Zhang H, Cui Z, Liang B, Jiang W, et al: Preoperative blood-routine markers and prognosis of esophageal squamous cell carcinoma: The Fujian prospective investigation of cancer (FIESTA) study. Oncotarget 8: 23841-23850, 2017.

5. Kim R, Keam B, Kwon D, Ock CY, Kim M, Kim TM, Kim HJ, Jeon YK, Park IK, Kang CH, et al: Programmed death ligand-1 expression and its prognostic role in esophageal squamous cell carcinoma. World J Gastroenterol 22: 8389-8397, 2016.

6. Li J, Li M, Gao F and Ge X: Serum microRNA-15a level acts as a potential diagnostic and prognostic biomarker for human esophageal squamous cell carcinoma. Cancer Biomark 18: 11-17, 2017.

7. Stein U, Walther W, Arlt F, Schwabe H, Smith J, Fichtner I, Birchmeier W and Schlag PM: MACC1, a newly identified key regulator of HGF-MET signaling, predicts colon cancer metastasis. Nat Med 15: 59-67, 2009.

8. Stein U, Smith J, Walther W and Arlt F: MACC1 controls Met: What a difference an Sp1 site makes. Cell Cycle 8: 2467-2469, 2009.

9. Shirahata A, Shinmura K, Kitamura Y, Sakuraba K, Yokomizo K, Goto T, Mizukami H, Saito M, Ishibashi K, Kigawa G, et al: MACC1 as a marker for advanced colorectal carcinoma. Anticancer Res 30: 2689-2692, 2010.

10. Ilm K, Fuchs S, Mudduluru G and Stein U: MACC1 is posttranscriptionally regulated by miR-218 in colorectal cancer. Oncotarget 7: 53443-53458, 2016.

11. Xia J, Wang H, Huang H, Sun L, Dong S, Huang N, Shi M, Bin J, Liao Y and Liao W: Elevated Orail and STIM1 expressions upregulate MACC1 expression to promote tumor cell proliferation, metabolism, migration, and invasion in human gastric cancer. Cancer Lett 381: 31-40, 2016.

12. Xie C, Wu J, Yun J, Lai J, Yuan Y, Gao Z, Li M, Li J and Song L: MACC1 as a prognostic biomarker for early-stage and AFP-normal hepatocellular carcinoma. PLoS One 8: e64235, 2013.

13. Qu JH, Chang XJ, Lu YY, Bai WL, Chen Y, Zhou L, Zeng Z, Wang CP, An LJ, Hao LY, et al: Overexpression of metastasisassociated in colon cancer 1 predicts a poor outcome of hepatitis B virus-related hepatocellular carcinoma. World J Gastroenterol 18: 2995-3003, 2012.

14. Tan W, Xie X, Li L, Tang H, Ye X, Chen L, Tang W, Gao J, Pan L, Zhang X, et al: Diagnostic and prognostic value of serum MACC1 in breast cancer patients. Oncotarget 7: 84408-84415, 2016.

15. Li H, Zhang H, Zhao S, Shi Y, Yao J, Zhang Y, Guo H and Liu X: Overexpression of MACC1 and the association with hepatocyte growth factor/c-Met in epithelial ovarian cancer. Oncol Lett 9: 1989-1996, 2015.

16. Sun L, Li G, Dai B, Tan W, Zhao H, Li X and Wang A: Silence of MACC1 expression by RNA interference inhibits proliferation, invasion and metastasis, and promotes apoptosis in U251 human malignant glioma cells. Mol Med Rep 12: 3423-3431, 2015. 
17. DeVorkin L, Hattersley M,Kim P, Ries J, Spowart J, Anglesio MS Levi SM, Huntsman DG, Amaravadi RK, Winkler JD, et al Autophagy inhibition enhances sunitinib efficacy in clear cell ovarian carcinoma. Mol Cancer Res 15: 250-258, 2017.

18. Zhan L, Zhang Y, Wang W, Song E, Fan Y, Li J and Wei B: Autophagy as an emerging therapy target for ovarian carcinoma. Oncotarget 7: 83476-83487, 2016.

19. Ahn JS, Ann EJ, Kim MY, Yoon JH, Lee HJ, Jo EH, Lee K, Lee JS and Park HS: Autophagy negatively regulates tumor cell proliferation through phosphorylation dependent degradation of the Notch1 intracellular domain. Oncotarget 7: 79047-79063, 2016.

20. Guo W, Wang H, Yang Y, Guo S, Zhang W, Liu Y, Yi X, Ma J, Zhao T, Liu L, et al: Down-regulated miR-23a contributes to the metastasis of cutaneous melanoma by promoting autophagy. Theranostics 7: 2231-2249, 2017.

21. Carchman EH, Matkowskyj KA, Meske L and Lambert PF: Dysregulation of autophagy contributes to anal carcinogenesis. PLoS One 11: e0164273, 2016

22. Jia YL, Xu M, Dou CW, Liu ZK, Xue YM, Yao BW, Ding LL, Tu KS, Zheng X and Liu QG: P300/CBP-associated factor (PCAF) inhibits the growth of hepatocellular carcinoma by promoting cell autophagy. Cell Death Dis 7: e2400, 2016.

23. Yue Z, Jin S, Yang C, Levine AJ and Heintz N: Beclin 1, an autophagy gene essential for early embryonic development, is a haploinsufficient tumor suppressor. Proc Natl Acad Sci USA 100: 15077-15082, 2003.
24. Qu X, Yu J, Bhagat G, Furuya N, Hibshoosh H, Troxel A, Rosen J, Eskelinen EL, Mizushima N, Ohsumi Y, et al: Promotion of tumorigenesis by heterozygous disruption of the beclin 1 autophagy gene. J Clin Invest 112: 1809-1820, 2003.

25. Weh KM, Howell AB and Kresty LA: Expression, modulation, and clinical correlates of the autophagy protein Beclin-1 in esophageal adenocarcinoma. Mol Carcinog 55: 1876-1885, 2016.

26. Lee E, Wei Y, Zou Z, Tucker K, Rakheja D, Levine B and Amatruda JF: Genetic inhibition of autophagy promotes p53 lossof-heterozygosity and tumorigenesis. Oncotarget 7: 67919-67933, 2016.

27. Lu C and Xie C: Radiation-induced autophagy promotes esophageal squamous cell carcinoma cell survival via the LKB1 pathway. Oncol Rep 35: 3559-3565, 2016.

28. Yamada E, Okada S, Bastie CC, Vatish M, Nakajima Y, Shibusawa R, Ozawa A, Pessin JE and Yamada M: Fyn phosphorylates AMPK to inhibit AMPK activity and AMP-dependent activation of autophagy. Oncotarget 7: 74612-74629, 2016.

29. He Y, Mo Q, Luo B, Qiao Y, Xu R, Zuo Z, Deng J, Nong X, Peng G, He W, et al: Induction of apoptosis and autophagy via mitochondria- and PI3K/Akt/mTOR-mediated pathways by E. adenophorum in hepatocytes of saanen goat. Oncotarget 7: 54537-54548, 2016. 